\title{
EFFECT OF BITE FORCE ON THE POWER SPECTRUM OF THE SURFACE ELECTROMYOGRAM OF HUMAN JAW MUSCLES
}

\author{
S. PAlla and M. M. ASH $\mathrm{J}_{\mathbf{R}}$ \\ Stomatognathic Physiology Laboratory, Department of Occlusion, Dental Research Institute, \\ School of Dentistry, University of Michigan, Ann Arbor, MI 48109, U.S.A.
}

\begin{abstract}
Summary-The frequency content of the surface electromyogram (EMG) of the anterior temporal and masseter muscles of 14 normal adults was obtained during maximum clench in intercuspal position, as well as at different bite force levels. Frequency analysis was carried out by computer using a fast Fourier transform algorithm to obtain the power-spectral density function and the power spectrum of the EMG signal. Bite force was monitored by a force transducer placed intra-orally. The power spectra of the anterior temporal muscle during maximun clench in intercuspal position were significantly wider than those of the masseter muscle, indicating that the EMG of the former muscle contained more power at high frequencies than that of the masseter muscle. Increase in bite force produced, in both muscles, a power-spectrum shift to lower frequencies. The shift was probably caused by recruitment of larger motor units and by increase in synchronization between them. Different contents of A, B and $C$ fibres and/or differences in thickness of skin covering the muscles could account for the differences between the temporal and masseter muscle power spectra.
\end{abstract}

\section{INTRODUCTION}

Electromyograms (EMG) can be analysed quantitatively in the time or frequency domain. Frequency analysis is carried out by a filter-bank analyser or by a digital computer using a fast Fourier transform algorithm to generate the power-spectral density function and the power spectra of the signal (Bendat and Piersol, 1971). Both systems have been used to investigate the frequency content of the EMG (Kadefors and Petersén, 1970; K watny, Thomas and Kwatny, 1970; Duxbury et al., 1975). In the last decade, more attention has been focused on frequency analysis, particularly because it offers a method of studying the motor-unit action potential in the EMG signal, even when this is obtained at high-contraction levels and the individual motor-unit action potentials are buried in the interference pattern. Indeed, the power spectrum of the EMG signal is directly related to the conduction velocity of the muscle-fibre action potential (Lindström, Magnusson and Petersén, 1970; Kadefors, Petersén and Broman, 1973) and therefore inversely to the average duration of the motor-unit action potentials composing the EMG signal (Kaiser and Petersén, 1963, 1965; Kadefors et al., 1973). Furthermore, the shape of the power spectrum is also determined by the degree of synchronization between motor units (Person and Libkind, 1970).

A common complaint of patients with functional disturbances of the masticatory system (dysfunctional patients) is muscle pain. Derijk, Jones and Keith (1977) reported two types of masseter-muscle EMG in dysfunctional patients: a high-frequency and a lowfrequency pattern. This observation suggested that EMG frequency analysis may help in understanding muscle dysfunction. However, before this technique can be used to study muscle function and dysfunc- tion, it is necessary to define the EMG power spectrum of the masticatory muscles of normal subjects, and to investigate how various parameters may affect it. One of these parameters is bite force. The study of the effect of bite force on the frequency content of the EMG is important because dysfunctional patients have a significantly lower maximal bite force than normal subjects (Molin, 1973; Helkimo, Carlson and Carmeli, 1975). Furthermore, the effect of the contraction level on the EMG frequency content has not been definitively established (Kwatny et al., 1970; Lindström et al., 1970; O'Donnel et al., 1973; Duxbury et al., 1975; Duxbury, Hughes and Clark, 1976).

Our purpose was to investigate, in normal subjects, the frequency content of the EMG signal of the temporal and masseter muscles during maximum clench in intercuspal position, as well as at different bite forces.

\section{MATERIALS AND METHODS}

\section{Subjects}

This study was of 14 dentulous subjects (12 male, 2 female) aged 21-35 yr. None gave a past or present history of functional disturbances of the masticatory system or of symptoms such as clicking, crepitation and pain in the mandibular joints, muscle pain or fatigue upon chewing, reduced jaw mobility, facial pain, headache and earache. No clinical examination was undertaken. Each subject gave informed consent.

\section{Clinical procedure}

Muscle activity was monitored by recording the EMG signals of both anterior temporal and both masseter muscles using bipolar silver electrodes (Grass E 4S, Grass Instruments, Quincy, Mass., U.S.A.) placed $2 \mathrm{~cm}$ apart in the direction of the 
muscle fibres, the position being determined by muscle palpation. After vigorously scrubbing the skin with an alcohol-soaked gauze pad, electrode cream (Grass EC2) was applied; adhesive collars were used to secure the electrodes. The earth electrode was attached to the right earlobe. The impedance of the active electrodes was always below $2 \mathrm{k} \Omega$ (Grass impedance meter EZM). The EMG signals were amplified differentially (Grass 7P511) and filtered between 10 and $1000 \mathrm{~Hz}$ (down $3 \mathrm{db}$ at 14 and $600 \mathrm{~Hz}$ ) to yield a flat frequency response between 25 and $320 \mathrm{~Hz}$. The amplified signals were recorded on paper (Grass EEG Polygraph 78), on magnetic tape (FM tape recorder Hewlett-Packard 3955; bandwith $0-1.25 \mathrm{kHz}$, Hewlett-Packard, Palo Alto, Ca 94304, U.S.A.) and digitized.

Bite force was measured in anterior-posterior, lateral and vertical direction with a miniature piezoelectric 3-component force transducer (Kistler 73631-b, Kistler Instruments, CH-8408 Winterthur, Switzerland) (Grassl, 1977). An upper and lower ticonium bar, fixed to the dental arches with acrylic resin, were used to position the transducer in the centre of the mouth, half the distance between the first molars. The transducer was fixed to the lower bar. Connection between upper bar and transducer was provided by a ball-and-socket joint placed in the middle of the transducer. The increase in vertical dimension was approximately $5 \mathrm{~mm}$ in the first molar region (for details see Palla et al., 1981). The amplified signals were recorded on paper and magnetic tape as described for the EMG signals.

The subject, sitting upright in a dental chair in a Faraday room, was instructed to clench for $3 \mathrm{~s}$ at maximum in intercuspal position, and to repeat the clench 10 times. Between clenches the subject rested for $40 \mathrm{~s}$. After $5 \mathrm{~min}$ of relaxation, the force transducer was inserted in the mouth and the subject was asked to clench maximally for $3 \mathrm{~s}$ and to repeat this 10 times. In order to avoid muscle fatigue, the subject relaxed for $40 \mathrm{~s}$ between clenches. Furthermore, the force transducer was removed after the 5 th clench for $1 \mathrm{~min}$ prior to continuing with the last 6 clenches. This procedure was repeated with the subject clenching on the force transducer at $1 / 2,1 / 4,1 / 8$ and $1 / 16$ of the maximal vertical force. To enable the subject to produce the desired bite force and to keep it constant during each clench, the signal of the vertical force component was displayed to him on a storage oscilloscope (Tektronix 5130N, Tektronix Inc., Beaverton, OR 97077, U.S.A.). No recordings at 1/16-maximum were taken on subject $\mathrm{KW}$.

\section{Data processing}

The EMG analogue signals were digitized at a sam pling rate of $1428 \mathrm{~Hz}$, recorded on magnetic tape and displayed on a graphics terminal for screening. For each clench, the beginning and end of the EMG signal were discarded, and only that portion at constant bite force was selected for analysis. The chosen portions were recorded sequentially on a new tape for subsequent frequency analysis. The procedure allowed editing of approximately $30 \mathrm{~s}$ of data for each bite condition. The detailed procedure for digitizing and editing was described by Geister, McCall and Ash (1975) and Brown et al. (1979).
EMG frequency analysis was carried out by computer using a fast Fourier transform (FFT) algorithm (Harding, 1976) to obtain the power-spectral density function (PSDF). The FFT was performed on 512 data points corresponding to $0.36 \mathrm{~s}$ of data. The resultant PSDF had a frequency range from 2.79 to $714.29 \mathrm{~Hz}$ and a resolution of $2.79 \mathrm{~Hz}$. However, frequencies below $27.90 \mathrm{~Hz}$ were discarded for the statistical analysis because the power of the first 10 points of the transform are of dubious value (Brown, 1975). Prior to the transformation, a straight-line fit was performed to remove any baseline offset or linear drift, and a cosine-bell window was applied. For each muscle and bite condition, we analysed $28.8 \mathrm{~s}$ of data by performing 80 FFTs and calculating the mean PSDF. This was found by computing the arithmetic mean of the power at the discrete frequencies for the ensemble of the smoothed PSDFs obtained from the transforms. The method was similar to that used by Brown (1975).

The PSDF values were listed on a high-speed line printer and stored on magnetic tape for further use, such as computation of the group mean PSDF and of the cumulative power function as well as plotting of the power spectra. The group mean PSDF was calculated for each muscle and bite condition by averaging the PSDFs of 13 subjects (the PSDFs of subject KW were not used in this computation). The power spectrum is a graphic display of the PSDF. The horizontal axis represents the frequency and the vertical axis the power (mean square value of the amplitude of the signal in a given frequency band). Before plotting, the PSDFs were smoothed by using a 5-point leastsquare curve-fitting technique (Savitzky and Golay, 1964) and normalized to their maximum value. The cumulative power function, $C P(f)$, represents the percentage of the total power below a discrete frequency. We calculated the frequencies at which $10,25,50,75$ and 90 per cent of the total power occurred $(F 10, F 25$, $F 50, F 75, F 90$ ) and the bandwiths between 10 and 90 and between 25 and 75 per cent of the total power $(B 10-90, B 25-75)$. These values enabled us to characterize the shape of the power spectra, and were used in the statistical tests.

In addition to the PSDF, we computed the root mean square (r.m.s.) voltage of the EMG signal for each muscle and bite condition. $28.8 \mathrm{~s}$ of data were divided in 41 samples of $0.7 \mathrm{~s}$ each, and the r.m.s. voltage was computed for each second sample. Mean r.m.s. voltage and standard deviation (SD) were calculated from these r.m.s. values. Any baseline offset was removed by subtracting the mean of the first sample from the value of each data point before computing the r.m.s. voltage.

The 3 bite force components were measured from the paper recordings to the nearest $0.1 \mathrm{~mm}$, converted to Newtons $(\mathrm{N})$ and the resultant bite force $\left(F_{r}\right)$ calculated (Palla et al., 1981). The measurements were performed twice for every clench, giving 22 values for each clench series. The values were averaged to determine mean $F_{r}$ and SD for each bite force.

\section{Statistical analysis}

Multivariate and univariate analysis of variance for repeated measures on the same subject (Rao, 1952; Winer, 1971) were used to test the null hypothesis of 
Table 1. Mean, SD and 95 per cent confidence interval (CI) of the frequencies in $\mathrm{Hz}$ at 10,50 and 90 per cent of the cumulative power. Maximum clench in intercuspal position (without transducer)

\begin{tabular}{ccccc}
\hline & & \multicolumn{3}{c}{ Per cent } \\
\cline { 3 - 5 } & Muscle & 10 & \multicolumn{2}{c}{50} \\
\hline \multirow{4}{*}{ Mean } & RAT & 61.03 & 144.94 & 280.37 \\
& LAT & 67.35 & 156.93 & 290.39 \\
& RM & 56.47 & 129.63 & 265.19 \\
SD & LM & 59.34 & 136.55 & 270.27 \\
& RAT & 12.430 & 39.132 & 53.205 \\
& LAT & 17.663 & 39.046 & 51.831 \\
& RM & 7.445 & 34.920 & 71.920 \\
CI & LM & 10.751 & 39.313 & 70.760 \\
& RAT & $53.85-68.21$ & $122.35-167.54$ & $249.65-311.09$ \\
& LAT & $57.15-77.55$ & $134.39-179.48$ & $260.46-320.32$ \\
& RM & $52.17-60.77$ & $109.47-149.80$ & $223.66-306.71$ \\
& LM & $53.13-65.55$ & $113.85-159.25$ & $229.41-311.12$ \\
& & & & \\
\hline
\end{tabular}

no statistically significant differences between the group mean PSDFs obtained for different bite conditions, for the 4 muscles and for the left and right side. With the Pearson product moment correlation coefficient (Steel and Torrie, 1960), we tested whether power spectra changes were linearly related to bite force. Wc used a multivariate Hotelling $T^{2}$ test (Winer, 1971) to determine if the EMG signal remained stationary during each clench series: the mean PSDF of the first $7.2 \mathrm{~s}$ of EMG signal was compared with that of the last $7.2 \mathrm{~s}$. All tests were performed at the 0.05 level of significance.

\section{RESULTS}

Power spectra obtained at maximum clench in intercuspal position

The mean frequencies at 10,50 and 90 per cent of the cumulative power for all subjects are given in Table 1, and the normalized-group mean power spectra of the left masseter and temporal muscle in Fig. 1. Visual comparison of these spectra reveals that the temporal-muscle EMG generally produced a wider spectrum than the masseter EMG. The difference was due to a more pronounced power decay of

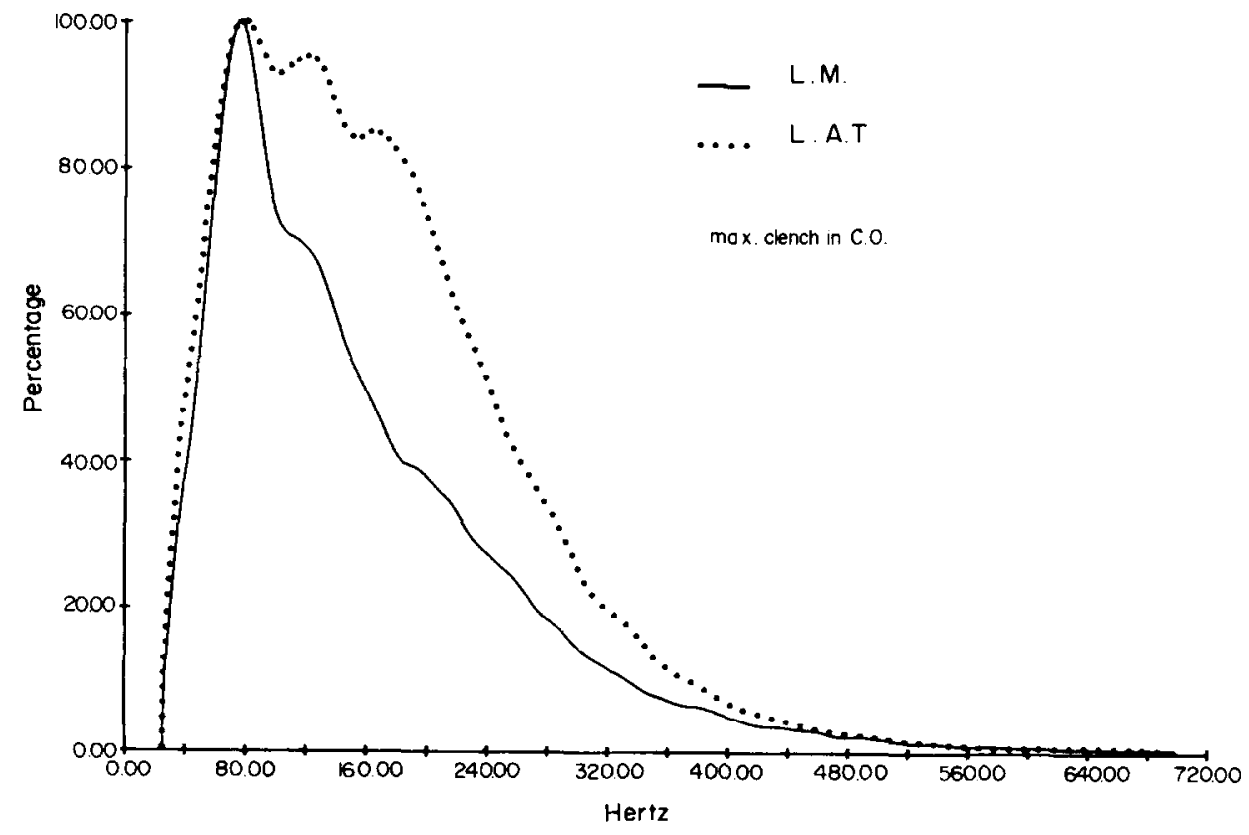

Fig. 1. Group mean power spectra of the left anterior temporal and masseter muscles EMG during maximum clench in intercuspal position (without force transducer). 
the masseter-muscle spectrum at frequencies higher than approx. $80 \mathrm{~Hz}$. Consequently, the EMG highfrequency components of this muscle were of smaller amplitude than those of the temporal muscle. Multivariate analysis of variance confirmed that the group mean power spectra of the temporal muscle were significantly wider than those of the masseter muscle $(p<0.025)$. No statistically significant difference was found among the left and right side for both muscles $(p<0.05)$.

The peak frequencies of the group mean power spectra were $64.17 \mathrm{~Hz}$ for the right and $78.12 \mathrm{~Hz}$ for the left temporal muscles; $75.34 \mathrm{~Hz}$ for the right and $78.13 \mathrm{~Hz}$ for the left masseter muscles.

\section{Effect of bite force on the PSDF}

The normalized-power spectra of the right masseter muscle showed that increasing the bite force produced a power spectrum shift to lower frequencies (Fig. 2). The most pronounced shift occurred between maxi- mum and half-maximum bite force. The mean frequency, F50, shifted continuously with decreasing bite force for both masseter muscles throughout 1/16maximum, for the right temporal muscle throughout 1/8-maximum and for the left temporal muscle throughout 1/4-maximum (Table 2). These findings indicated that the frequency of the surface EMG decreased with increasing bite force.

A 4-factor multivariate analysis of variance proved that the group mean power spectra obtained at different bite forces varied signiticantly $(p<0.0005)$. However, bite force did not have the same effect on all the subjects, nor on the two muscles (significant interactions force-subject and force-muscle: $p<0.0005$ ). Indeed, Fig. 3 shows the individual pattern in the variation of the $F 50$ at different force levels for the right masseter muscle. The F50 of the left masseter and of both temporal muscles exhibited similar variations. Since the correlation coefficients between the frequencies at the $10,25,50,75$ and 90 per cent of the


Fig. 2. Spectral modifications due to bite force. Each spectrum is the group mean (13 subjects) at a given force level (RM: right masseter). 
Table 2. Frequency in $\mathrm{Hz}$ at 10 and 50 per cent of the cumulative power for each muscle and force level; 14 subjects

\begin{tabular}{|c|c|c|c|c|c|}
\hline \multirow[b]{2}{*}{ Muscle } & \multirow[b]{2}{*}{ Force } & \multicolumn{2}{|c|}{$F 10$} & \multicolumn{2}{|c|}{$F 50$} \\
\hline & & Mean & SD & Mean & SD \\
\hline \multirow[t]{5}{*}{ RAT } & $\operatorname{Max}$ & 54.05 & 10.613 & 121.76 & $31.256(1)$ \\
\hline & $1 / 2$ & 60.71 & 12.794 & 143.89 & $27.209(2)$ \\
\hline & $1 / 4$ & 62.96 & 10.673 & 148.61 & $22.565(3)$ \\
\hline & $1 / 8$ & 64.38 & 9.210 & 149.93 & $18.189(4)$ \\
\hline & $1 / 16$ & 61.74 & 8.144 & 144.72 & $16.610(5)$ \\
\hline \multirow{5}{*}{ LAT } & Max & 59.29 & 11.058 & 133.49 & $30.698(1)$ \\
\hline & $1 / 2$ & 65.40 & 13.422 & 152.09 & $26.527(2)$ \\
\hline & $1 / 4$ & 67.82 & 13.021 & 155.94 & $23.859(3)$ \\
\hline & $1 / 8$ & 66.99 & 9.854 & 153.47 & $20.971(4)$ \\
\hline & $1 / 16$ & 59.06 & 6.420 & 141.07 & $18.135(5)$ \\
\hline \multirow[t]{5}{*}{ RM } & $\operatorname{Max}$ & 52.70 & 7.378 & 107.92 & $33.456(1)$ \\
\hline & $1 / 2$ & 53.88 & 8.749 & 127.96 & $28.775(2)$ \\
\hline & $1 / 4$ & 55.11 & 9.111 & 132.64 & $24.987(3)$ \\
\hline & $1 / 8$ & 56.99 & 8.598 & 136.56 & $23.553(4)$ \\
\hline & $1 / 16$ & 58.02 & 9.309 & 137.04 & $24.756(5)$ \\
\hline \multirow[t]{5}{*}{ LM } & Max & 55.04 & 8.857 & 113.66 & $34.005(1)$ \\
\hline & $1 / 2$ & 55.34 & 10.530 & 130.42 & $29.382(2)$ \\
\hline & $1 / 4$ & 57.57 & 9.295 & 138.84 & $24.339(3)$ \\
\hline & $1 / 8$ & 58.83 & 9.500 & 140.35 & $23.074(4)$ \\
\hline & $1 / 16$ & 59.61 & 10.033 & 143.86 & $21.423(5)$ \\
\hline
\end{tabular}

Significance: (1) versus (2) $p<0.01$; (1) versus (3) $p<0.01$; (1) versus (4) $p<0.01$; (1) versus (5) $p<0.01$.
Table 3. Correlation coefficients between the frequencies at the 10,25, 50 and 90 per cent of the cumulative power and $B 10-90, B 25-75$

\begin{tabular}{lcccccc}
\hline$B 10-90$ & 0.98 & & & & & \\
$F 10$ & 0.65 & 0.69 & & & & \\
$F 25$ & 0.77 & 0.81 & 0.97 & & & \\
$F 50$ & 0.88 & 0.90 & 0.91 & 0.98 & & \\
$F 75$ & 0.94 & 0.95 & 0.86 & 0.94 & 0.99 & \\
$F 90$ & 0.96 & 0.99 & 0.80 & 0.89 & 0.95 & 0.99 \\
& $B 25-75 B 10-90$ & $F 10$ & $F 25$ & $F 50$ & $F 75$ \\
\hline
\end{tabular}

$C P(f)$ and the bandwiths $B 10-90$ and $B 25-75$ were very high (Table 3), any of these frequencies can be used to statistically characterize variations in the power spectra shape. The $F 50$ varied significantly at different bite forces $(p<0.0005)$, but only the differences between maximum and $1 / 2$, maximum and $1 / 4$, maximum and $1 / 8$ and maximum and $1 / 16$ were significant (Newman-Keuls test; $p<0.01$ ) (Table 2). As indicated in Figs 2 and 3, the main frequency shift occurred between maximum clench and $1 / 2$. However, the shift continued, although far less pronounced, at lower forces for most subjects. In 37 of the 52 muscles examined, the shift was present throughout $1 / 8$-maximum. Furthermore, the individual power spectra indicated that the shift concerned primarily frequencies above approx. $80 \mathrm{~Hz}$.

The correlation coefficients calculated between the $F 10$ and $F 50$ values and r.m.s. voltage confirmed the dependence of power-spectra shape on bite-force
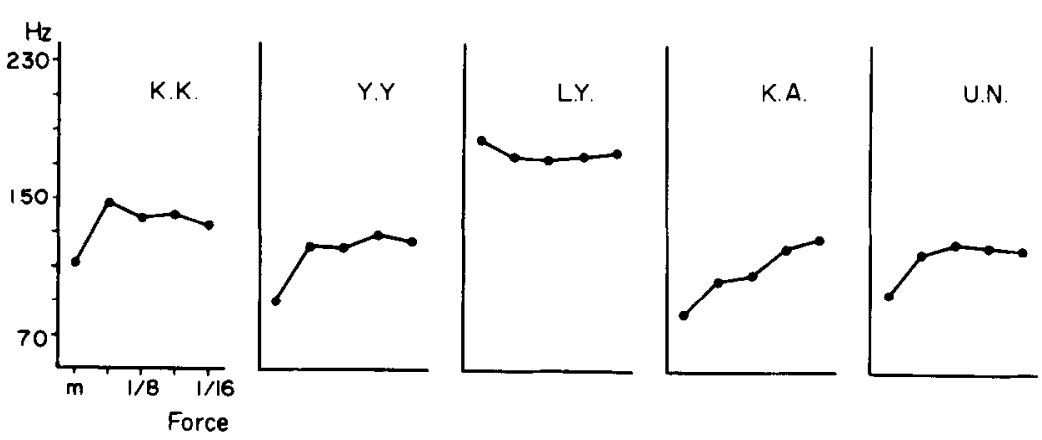

RM
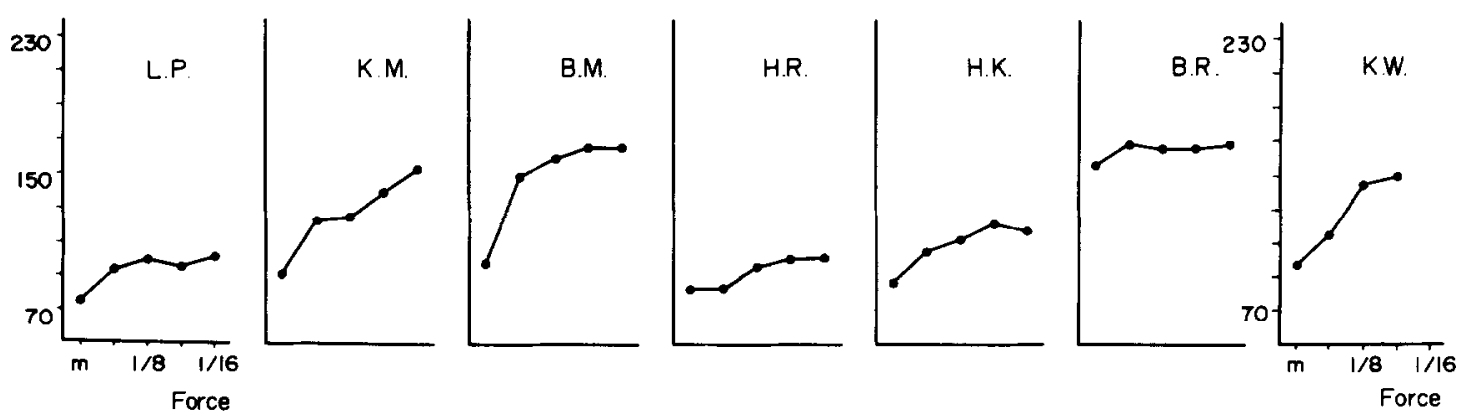

Fig. 3. Individual variations of the frequency at the 50 per cent of the cumulative power $[C P(f)]$ due to bite force. Same scale for all subjects. 


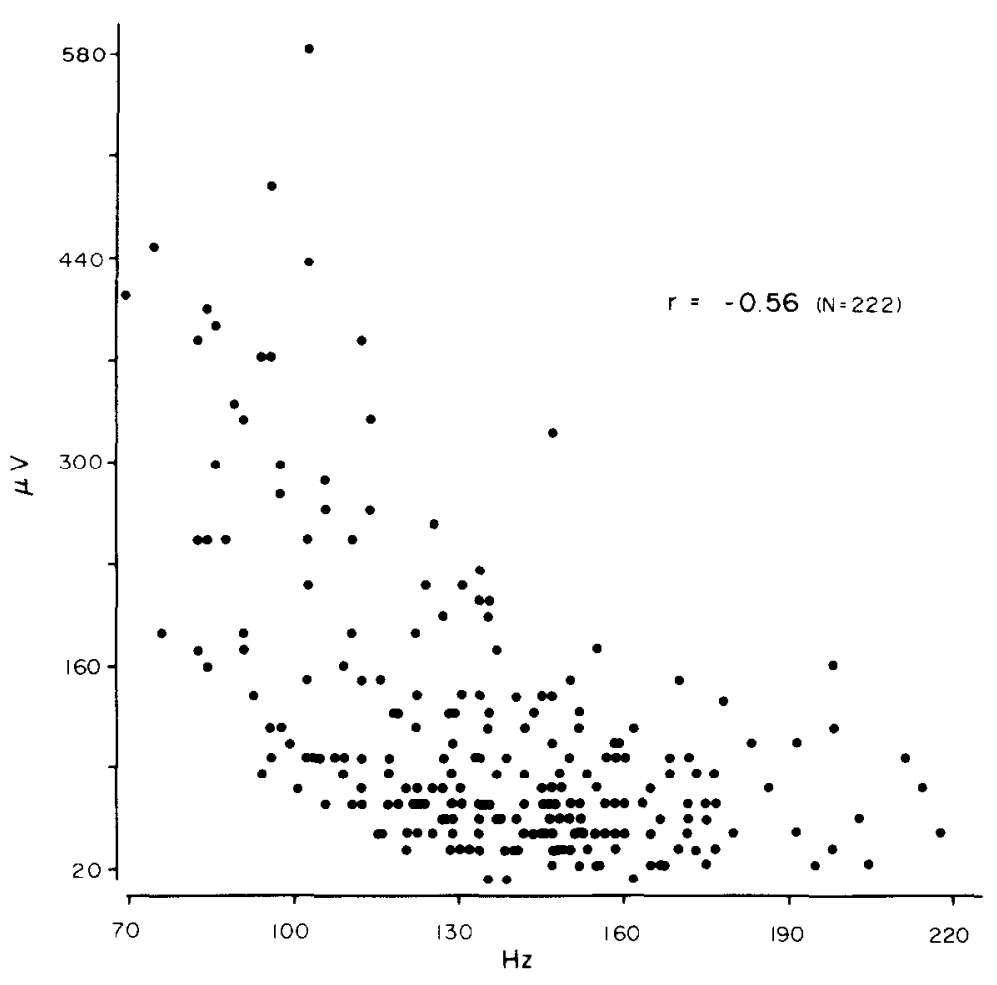

Fig. 4. Scatter plot: r.m.s. voltage versus F50. Data of 4 muscles and 14 subjects throughout 1/8-maximum.

level. The correlation coefficients calculated for each subject and each muscle throughout 1/8-maximum are given in Table 4 . The mean and 95 per cent confidence interval calculated from all the individual coefficients were $-0.92(-0.86,-0.95)$ for $F 50$ and $-0.69(-0.52,-0.80)$ for $F 10$. The group correlation coefficient computed by pooling the values obtained from the 4 muscles and 14 subjects throughout 1/8-maximum were -0.56 for $F 50$ and -0.36 for $F 10$ $(n=222)$; they were significantly different from zero $(p<0.0005)$. The moderate degree of correlation between $F 50$ and r.m.s. voltage is visible also from the scatter plot of Fig. 4. The correlation coefficients were higher between $F 50$ and r.m.s. voltage than between F10 and r.m.s. voltage, reflecting a greater influence of bite force on the high- rather than on the lowfrequency content of the EMG signal.

Table 4 and Fig. 3 indicate that the power spectra of a few muscles demonstrated a tendency to shift at random, or towards a lower frequency range with de-

Table 4. Individual correlation coefficients between the frequencies at $10 / 50$ per cent of the cumulative power and the r.m.s. voltage for the 4 muscles calculated throughout $1 / 8$-maximum

\begin{tabular}{|c|c|c|c|c|c|c|c|c|}
\hline \multirow[b]{2}{*}{ Subject } & \multicolumn{4}{|c|}{$F 10$} & \multicolumn{4}{|c|}{$F 50$} \\
\hline & RAT & LAT & RM & $\mathrm{LM}$ & RAT & LAT & $\mathrm{RM}$ & LM \\
\hline KK & -0.81 & -0.97 & -0.82 & -0.76 & -0.85 & -0.92 & -0.89 & -0.98 \\
\hline YY & -0.96 & -0.11 & +0.88 & +0.98 & -0.99 & -0.99 & -0.99 & -0.99 \\
\hline LY & -0.53 & +0.51 & -0.78 & +0.20 & -0.38 & +0.21 & +0.93 & +0.06 \\
\hline $\mathrm{KA}$ & -0.97 & -0.91 & -0.23 & +0.51 & -0.99 & -0.97 & -0.95 & -0.99 \\
\hline UN & -0.77 & -0.99 & +0.32 & +0.83 & -0.99 & -0.99 & -0.99 & -0.99 \\
\hline $\mathrm{RC}$ & +0.29 & +0.85 & -0.34 & -0.67 & +0.18 & +0.65 & +0.65 & -0.28 \\
\hline LP & -0.91 & -0.99 & -0.41 & -0.81 & -0.98 & -0.99 & -0.99 & -0.92 \\
\hline $\mathrm{KM}$ & -0.99 & -0.87 & -0.97 & -0.91 & -0.96 & -0.80 & -0.99 & -0.98 \\
\hline $\mathrm{BM}$ & -0.93 & -0.97 & -0.99 & -0.90 & -0.92 & -0.99 & -0.99 & -0.99 \\
\hline IIR & -0.46 & -0.21 & +0.83 & +0.14 & -0.99 & -0.91 & -0.76 & -0.82 \\
\hline HK & -0.98 & -0.99 & -0.14 & -0.68 & -0.98 & -0.96 & -0.96 & -0.98 \\
\hline BR & -0.39 & -0.30 & -0.93 & -0.90 & -0.02 & +0.39 & -0.78 & -0.48 \\
\hline IIJ & -0.98 & -0.52 & -0.59 & -0.65 & -0.99 & -0.47 & -0.98 & -0.97 \\
\hline KW & -0.89 & +0.34 & -0.85 & & -0.91 & +0.79 & -0.97 & \\
\hline
\end{tabular}



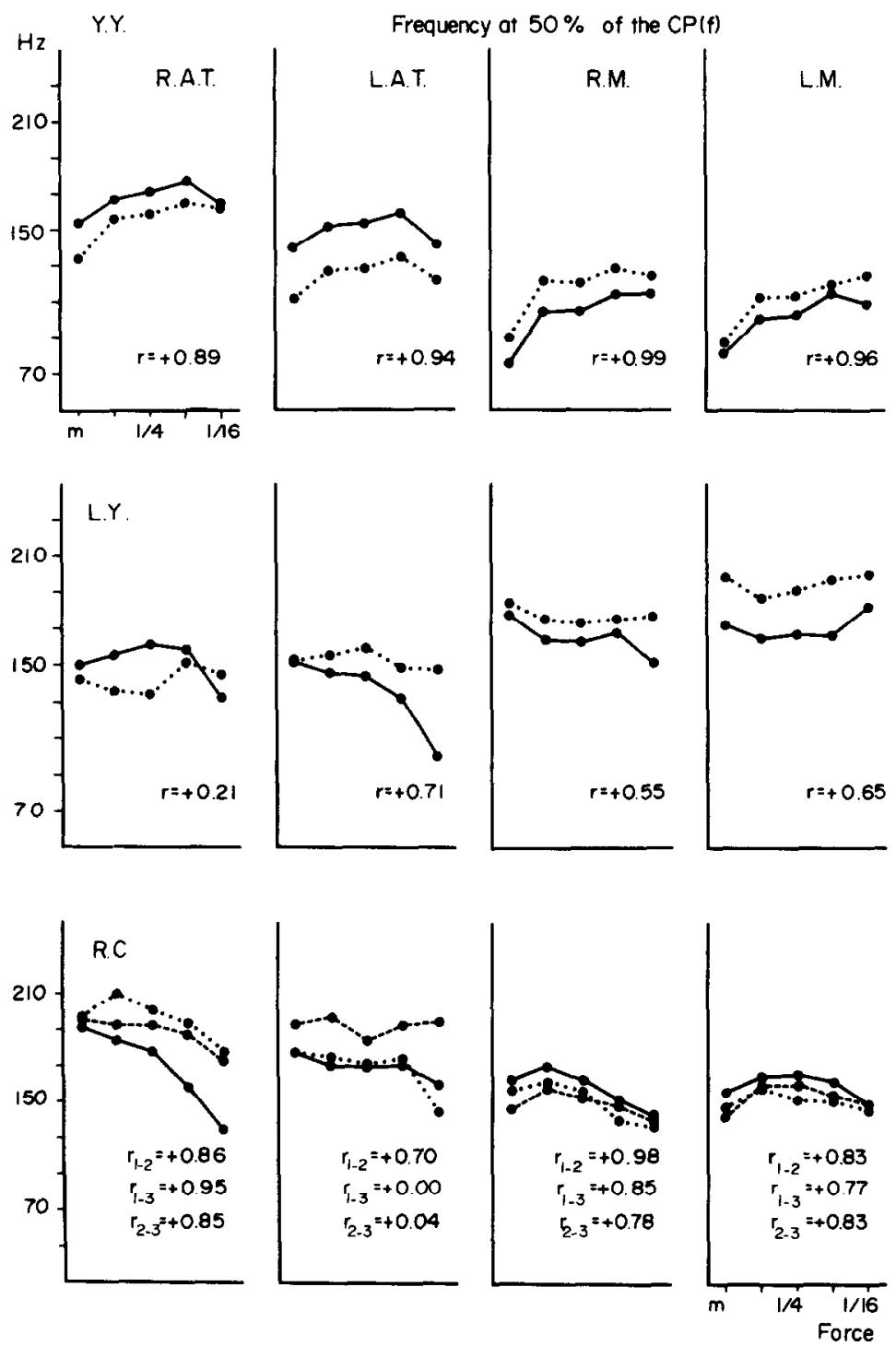

Fig. 5. Graphs and correlation coefficients for test-retest runs.

creasing bite force. This phenomenon was evident in subjects BR, LY and RC. The left temporal muscle of subject $R C$ and the left and right masseter and right temporal muscle from subject LY did not even show the high frequency shift with decreasing bite force from maximum to $1 / 2$-maximum. It is interesting to note that with reference to maximal bite force, these 2 subjects had by far the lowest values (RC $381.40 \mathrm{~N}$; LC $580.22 \mathrm{~N}$ ). The mean maximum bite force for the 14 subjects was $770.35 \pm 190.40 \mathrm{~N}$ (95 per cent confidence interval $660.41-880.28 \mathrm{~N}$ ).

\section{Effect of hite force related to time.}

In 2 subjects, bite force experiments were repeated 1 week later, and in a 3rd subject after 1 week and after $1 \mathrm{yr}$. Although the shape of the power spectra varied between sessions, bite-force variations produced similar power spectra changes in all sessions. The correlation coefficients for test-retest runs calculated between the $F 50$ values at different sessions are given in Fig. 5.

\section{Stationarity of the EMG signal}

In order to compare the stationarity of the EMG signal throughout each bite condition, the group mean power spectrum obtained from the first $7.2 \mathrm{~s}$ of EMG was compared with that from the last $7.2 \mathrm{~s}$. Statistically significant differences were found only for the power spectra of both masseter muscles during maximum clench in intercuspal position. The group mean power spectrum at the beginning was wider than that at the end of the clench series $(p<0.05)$.

\section{DISCUSSION}

Increasing bite force produced a power-spectrum shift to lower frequencies. The results agree with the findings of Duxbury et al. $(1975,1976)$ for the masseter muscle and by Lindström et al. (1970) for other striated muscles. The width of the power spectrum is related inversely to the average duration of the motor-unit action potentials (MUAP) composing the EMG signal (Cenkovich and Gersten, 1963; Kaiser 
and Petersén, 1963, 1965; Kadefors et al., 1973). Force augmentation is produced by an increase in the motoneuron firing rate and by recruitment of new motor units (De Luca, 1979). Motor units are activated according to their size, the recruitment order being from small to large (De Luca, 1979). This was also confirmed for the masseter muscle (Yemm, 1977; Goldberg, 1978). As the duration of the MUAP seems to be related to the size of a motor unit (Buchtal, Guld and Rosenfalk, 1955), the recruitment of larger motor units at higher bite forces may have increased the average duration of the MUAPs and therefore produced the power-spectrum shift to lower frequencies.

Motor-unit recruitment is the dominant mechanism for force increase up to 30 per cent maximal voluntary contraction (MVC) and plays only a secondary role at higher force levels, particularly above 75 per cent MVC (De Luca, 1979). We found the most pronounced power-spectra shift at force levels above 50 per cent of maximum clench. Therefore, it is unlikely that this shift was caused solely by recruitment of larger motor units. Another mechanism which may have caused the power-spectrum shift is an increased synchronization of the motor units, as this increases the duration of the interference EMG fluctuation (Person and Libkind, 1970). Motor units tend to synchronize with increasing muscle contraction (Person and Kudina, 1968), and preliminary results confirmed that this phenomenon can also occur in the masseter muscle (S. Palla, unpublished). Thus, the power-spectrum shift to lower frequencies with increasing bite force can be attributed both to recruitment of motor units with action potentials of longer duration, and to increased synchronization between motor units.

Mortimer. Magnusson and Petersén (1970) showed, in the cat, that the conduction velocity of the action potential along the muscle fibre decreases under ischemia because of accumulation of metabolic byproducts. This velocity change results in an increased duration of the action potential. Blood-flow studies in the masticatory muscles (Bonde-Petersen and Christensen, 1973; Rasmussen et al., 1977), although inconclusive, suggested that blood flow decreases during maximal contraction. Nilsson and Ingvar (1967) reported that tetanic stimulation of the anterior tibial muscle at $50 \mathrm{~Hz}$ produced an initial increase in blood flow followed by total arrest. Because in our study clenches were short, an initial increase in blood flow may have occurred during clenching, excluding an accumulation of metabolic by-products even at maximum clench. Moreover, the clenches were spaced with long rest periods, during which reactive hyperaemia occurs (Rasmussen et al., 1977),

The power spectra of 3 subjects shifted at random or towards a higher frequency range with increasing bite force. Repetition of the experiments in 2 of these 3 subjects at different sessions showed the same power-spectra variations. This indicated actual physiologic changes. Four muscles of these 2 subjects did not show a shift to higher frequencies with decreasing bite force from maximum to 1/2-maximum. These 2 subjects had maximum bite forces far below the group mean. The increase in bite force from $1 / 2$ to maximum was similar to that of the other subjects, but the
EMG-r.m.s. voltage increase was approximately 22 per cent below the group mean. As an increase in the synchronization of motor units reflects itself in an increase in the integrated electrical activity (Person and Libkind, 1970), it is possible that synchronization of motor units did not increase as much, or did not have the same effect on the EMG as in the muscles of the other subjects. The results of a preliminary study indicated that synchronization of motor units does not increase in all subjects after varying bite force from 1/2 to maximum (S. Palla, unpublished). Furthermore, out-of-phase synchronization is necessary to increase the duration of the EMG interference-pattern fluctuations (Person and Libkind, 1970). A power-spectrum shift to higher frequencies with increasing muscle contraction was reported for 2 hand muscles by Kwatny et al. (1970). However, they did not specify whether the highest force level corresponded to the maximal voluntary contraction. Hence, a comparison of their results with ours is not possible.

Except for 4 out of the 56 muscles, the spectra obtained at maximum clench in intercuspal position were wider, and had a higher $F 50$ value, than those obtained at maximum clench on the transducer. Sato (1966) reported a power-spectrum shift to lower frequencies as a function of muscle lengthening. Thus, the presence of the force transducer, which lengthened the elevators, could have accounted for the difference in the power spectra obtained at maximum clench with and without transducer.

The power spectra obtained during maximum clench in intercuspal position had 90 per cent of the power below approximately $290 \mathrm{~Hz}$. Although the shape of the power spectra and, therefore, the frequency content of the surface EMG also depends upon technical factors (Schanne and Chaffin, 1970; Zipp, 1978), the overall shape of the spectra and the frequency range of the main power of the masseter muscle corresponded to that reported previously (Brown, 1975; Duxbury et al., 1975, 1976). No comparable data were found in the literature for the temporal muscle. The difference in the frequency content of the EMG of the masseter and temporal muscles is not surprising, as differences in the power-spectra width were reported also for other striated muscles (Kaiser and Petersén, 1963; Herberts, 1969; Kadefors and Petersén, 1970). The greater overall high-frequency content of the temporal-muscle EMG may reflect the different amount of $\mathrm{A}, \mathrm{B}$ and $\mathrm{C}$. fibres in the 2 muscles (Taylor, 1976). The temporal muscle, the major postural muscle of the mandible (Møller, 1966) contains more $B$ and C fibres than the masseter (Taylor, 1976). These muscle fibres form motor units of smaller size than A fibres (Taylor, 1976), and therefore generate MUAPs of relatively short duration. Herberts (1969) reported that the soleus muscle of the leg had a lower low-frequency content than other striated muscles. The soleus is composed uniformly of type-B fibres (Ezaguirre and Fidone, 1975). The different shape of the masseter- and temporalmuscles power spectra may be caused in part also by the different thicknesses of the soft tissues covering the 2 muscles, because high skin-resistance lowers the high-frequency content of the EMG (Schanne and Chaffin, 1970; Blinowska and Piotrkiewicz, 1978). 
Acknowledgements-We wish to thank Mr P. Steusloff and Dr Y. Yamada for their help in developing the computer programs. This work was supported by U.S. Health Department Grant DE 02731-12.

Address for reprints request: Dr Sandro Palla, Zahnärztliches Institut, Plattenstr. 11, CH-8032 Zürich, Switzerland.

\section{REFERENCES}

Bendat J. S. and Piersol A. G. 1971. Random Data: Analysis and Measurements Procedures. Wiley-Interscience, New York.

Blinowska K. and Piotrkiewicz M. 1978. A study of surface electromyograms by means of digital simulation. II Spectral analysis of simulated and experimental electromyograms. Electromyogr. clin. Neurophysiol. 18, 95-105.

Bonde-Petersen F. and Christensen V. L. 1973. Blood flow in human temporal muscle during tooth grinding and clenching as measured by ${ }^{133}$ xenon clearance. Scand. $J$. dent. Res. 81, 272-275.

Brown G. S. 1975. The fidelity of digital recordings. A framework for future decisions. Thesis, University of Michigan, Ann Arbor.

Brown G. S., Altug S. S., McCall W. D. and Geister D. E. 1979. Software support for computerized electromyography in clinical dentistry. IEEE Trans. biomed. Engng 26, 357-365.

Buchthal F., Guld C. and Rosenfalk P. 1955. Innervation zone and propagation velocity in human muscle. Acta physiol scand. 35, 174-190.

Cenkovich F. S. and Gersten J. W. 1963. Fourier analysis of the normal human electromyogram. Am. J. phys. Med. 42, 192-204.

De Luca C. J. 1979. Physiology and mathematics of myoelectric signals. IEEE Trans. biomed. Enyng 26, 313-325.

Derijk W. G., Jones G. L. and Keith K. D. 1977. Myographic characteristics and patient reported pain in patients with TMJ dysfunction. J. dent. Res. 56, Special Issue A. Abstract 193.

Duxbury A. J., Hughes D. F., Clark D. E. and Sharpe T. C. 1975. The bandwith of masseter electromyograms from bipolar surface electrodes. Electromyogr. clin. Neurophysiol. 15, 209-221.

Duxbury A. J., Hughes D. F. and Clark D. E. 1976. Power spectral distribution of the masseter electromyogram from surface electrodes. J. oral Rehab. 3, 333-339.

Ezaguirre C. and Fidone S. J. 1975. Physiology of the Nervous System, 2nd edn, p. 217. Ycar Book Mcdical Publishers Inc., Chicago.

Geister D. E., McCall W. D. and Ash M. M. 1975. Computerized data acquisition and analysis for real-time electromyography in clinical dentistry. Proc. IEEE 63, 1404-1414.

Goldberg L. J. 1978. Twitch tension, spike amplitude and recruitment order of masseter muscle single motor units. In: Oral Physiology and Occlusion (Edited by Perryman J. H.) pp. 78-90. Pergamon Press, New York.

Grassl H. 1977. Ein neues piezoelektrisches MiniaturDrei-Komponenten - Kraftmesselement. Messen Prüfen 9, $536-542$.

Harding L. 1976. Numerical analysis and applications soft ware abstracts. Computing Center memo 341, 2nd edn. University of Michigan, Computing Center, Ann Arbor, Mich.

Helkimo E., Carlson G. E. and Carmeli Y. 1975. Bite force in patients with functional disturbances of the masticatory system. J. oral Rehab. 2, 397-406.

Herberts P. 1969. Myoelectric signals in control of prostheses. Acta orthop. scand. Suppl. 124.

Kadefors R. and Petersén I. 1970. Spectral analysis of myoelectric signals from muscles of the pelvic floor during voluntary contraction and during reflex contrac- tions connected with ejaculation. Electromyography 10 , 45-68.

Kadefors R., Petersén I. and Broman H. 1973. Spectral analysis of events in the electromyogram. New Dev. Electrom ogr. clin. Neurophysiol. 1, 628-637.

Kaiser E. and Petersén I. 1963. Frequency analysis of muscle action potentials during tetanic contraction. Electromyography 3, 5-17.

Kaiser E. and Petersén I. 1965. Muscle action potentials studied by frequency analysis and duration measurements. Acta neurol. Scand. 41, Suppl. 13, 213-236.

Kwatny E., Thomas D. H. and Kwatny H. G. 1970. An application of signal processing techniques to the study of myoelectric signals. IEEE Trans. biomed. Engng 17, 303-313.

Lindström L., Magnusson R. and Petersén I. 1970. Muscular fatigue and action potential conduction velocity changes studied with frequency analysis of EMG signals. Electromyography 10, 341-356.

Molin C. 1973. Studies in mandibular pain dysfunction syndrome. Swedish dent. J. 66, Suppl. 4.

Moller E. 1966. The chewing apparatus. An electromyographic study of the action of the muscles of mastication and its correlation to facial morphology. Acta physiol. scand. 69, Suppl. 280.

Mortimer J. T., Magnusson R. and Petersén I. 1970. Conduction velocity in ischemic muscle: effect on EMG frequency spectrum. Am. J. Physiol. 219, 1324-1329.

Nilsson B. and Ingvar D. H. 1967. Intramuscular pressure and contractile strength related to muscle blood flow in man. Scand. J. clin. Lab. Invest. 19, Suppl. 93, 31-38.

O’Donnel R. D., Rapp J., Berkhout J. and Adey W. R 1973. Autospectral and coherence patterns from two locations in the contracting biceps. Electromyogr. clin. Neurophysiol. 13, 259-269.

Palla S., Bailey J. O., Grassl H. and Ash M. M. 1981. The effect of bite force on the duration and latency of the menton tap silent period. J. oral Rehab. In press.

Person R. S. and Kudina L. P. 1968. Cross-correlation of electromyograms showing interference patterns. Electroenceph. clin. Neurophysiol. 25, 58-68.

Person R. S. and Libkind M. S. 1970. Simulation of electromyograms showing interference patterns. Electroenceph. clin. Neurophysiol. 28, 625-632.

Rao C. K. 1952. Advanced Statistical Methods in Biometric Research. Wiley, New York.

Rasmussen O. C., Bonde-Petersen F., Christensen L. V. and Mollcr E. 1977. Blood flow in human mandibular elevators at rest and during controlled bite. Archs oral Biol. 22, 539-553.

Sato M. 1966. A problem in the frequency analysis of the electromyogram. Electromyography 6, 21-23.

Savitzky A. and Golay M. J. E. 1964. Smoothing and differentiation of data by simplified least squares procedures. Analyt. Chem. 36, 1627-1639.

Schanne F, J. and Chaffin D. B. 1970. The effects of skin resistance and capacitance coupling on EMG amplitude and power spectra. Electromyography 10, 273-286.

Steel R. G. and Torrie J. H. 1960. Principles and Procedures of Statistics, p. 183. McGraw-Hill, New York.

Taylor A. 1976. Fibre types in the muscles of mastication In: Mastication (Edited by Anderson D. J. and Matthews B.) pp. 16-24. Wright, Bristol.

Zipp P. 1978. Effect of electrode parameters on the bandwidth of the surface e.m.g. power-density spectrum. Med Biol. Engng Comput. 16, 537-541.

Winer B. J. 1971. Statistical Principles in Experimental Design. 2nd edn. McGraw-Hill, New York.

Yemm R. 1977. The orderly recruitment of motor units of the masseter and temporal muscles during voluntary isometric contraction in man. J. Physiol., Lond. $\mathbf{2 6 5}$. 163-174. 